\title{
Surface Structures and Thermal Desorption Behaviors of Cyclopentanethiol Self-Assembled Monolayers on Au(111)
}

\author{
Hungu Kang, Youyoung Kim, Taesun Park, Joon B. Park, ${ }^{\dagger, *}$ Eisuke Ito,${ }^{\ddagger}$ Masahiko Hara, ${ }^{\ddagger}$ and Jaegeun Noh $^{*}$ \\ Department of Chemistry, Hanyang University, Seoul 133-791, Korea. E-mail: jgnoh@hanyang.ac.kr \\ ${ }^{\dagger}$ Institute of Fusion Science, Department of Chemistry Education, Chonbuk National University, Jeonbuk 561-756, Korea \\ E-mail: joonbumpark@jbnu.ac.kr \\ ${ }^{\ddagger}$ Flucto-order Functions Research Team, RIKEN-HYU Collaboration Center, RIKEN, 2-1 Hirosawa, Wako, \\ Saitama 351-0198, Japan

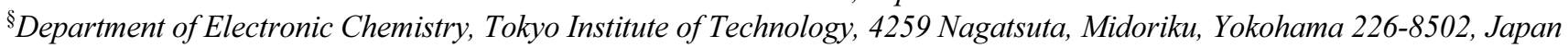 \\ Received January 3, 2011, Accepted February 15, 2011
}

\begin{abstract}
The surface structures, adsorption conditions, and thermal desorption behaviors of cyclopentanethiol (CPT) self-assembled monolayers (SAMs) on $\mathrm{Au}(111)$ were investigated by scanning tunneling microscopy (STM), X-ray photoelectron spectroscopy (XPS), and thermal desorption spectroscopy (TDS). STM imaging revealed that although the adsorption of $\mathrm{CPT}$ on $\mathrm{Au}(111)$ at room temperature generates disordered SAMs, CPT molecules at $50{ }^{\circ} \mathrm{C}$ formed well-ordered SAMs with a $(2 \sqrt{3} \times \sqrt{5}) \mathrm{R} 41^{\circ}$ packing structure. XPS measurements showed that CPT SAMs at room temperature were formed via chemical reactions between the sulfur atoms and gold surfaces. TDS measurements showed two dominant TD peaks for the decomposed fragments $\left(\mathrm{C}_{5} \mathrm{H}_{9}{ }^{+}\right.$, $\mathrm{m} / \mathrm{e}=69)$ generated via $\mathrm{C}-\mathrm{S}$ bond cleavage and the parent molecular species $\left(\mathrm{C}_{5} \mathrm{H}_{9} \mathrm{SH}^{+}, \mathrm{m} / \mathrm{e}=102\right)$ derived from a recombination of the chemisorbed thiolates and hydrogen atoms near $440 \mathrm{~K}$. Interestingly, dimerization of sulfur atoms in $n$-alkanethiol SAMs usually occurs during thermal desorption and the same reaction did not happen for CPT SAMs, which may be due to the steric hindrance of cyclic rings of the CPT molecules. In this study, we demonstrated that the alicyclic ring of organic thiols strongly affected the surface structure and thermal desorption behavior of SAMs, thus providing a good method for controlling chemical and physical properties of organic thiol SAMs.
\end{abstract}

Key Words : Cyclopentanethiol, Self-assembled monolayers, Scanning tunneling microscopy, Thermal desorption spectroscopy, Structure

\section{Introduction}

Self-assembled monolayers (SAMs) of organic thiols on metal surfaces have drawn considerable attention for more than 20 years because they offer an excellent way of generating functional organic thin films with high degrees of structural order and chemical stability for a wide range of applications, such as corrosion inhibition, chemical and biosensing, nanopatterning, and molecular electronics. ${ }^{1-4}$ To date, simple alkanethiol SAMs on gold provide the best model system for elucidating self-assembled mechanisms, packing structure, interface structure, and thermal stability. ${ }^{1-10}$ Alkanethiols at saturation coverage form a closely packed $(\sqrt{3} \times \sqrt{ } 3) \mathrm{R} 30^{\circ}$ structure or a $\mathrm{c}(4 \sqrt{3} \times 2 \sqrt{ } 3) \mathrm{R} 30^{\circ}$ superlattice (often denoted as a $\mathrm{c}(4 \times 2)$ superlattice). ${ }^{3,6,7,10}$ Several different two-dimensional (2D) phases were observed depending on the surface coverage and preparation conditions. ${ }^{3,5}$ 2D SAMs were formed by a delicate balance between sulfur atom-gold substrate interaction and molecule-molecule interaction. ${ }^{1}$ In particular, the backbone structures of organic thiols strongly affect the structural order and 2D packing structures of SAMs.

Recently, SAMs formed by diamondoid thiol derivatives with various bulky hydrocarbon cages have drawn much interest because they provide an unique molecular template for creating functional layers or nanopatterning with high aspect ratios. ${ }^{11-15}$ For instance, the adsorption of adamantanethiols on $\mathrm{Au}(111)$ yields the formation of highly crystalline ordered SAMs with multiple lattice structures with $(7 \times 7)$ and $(\sqrt{ } 91 \times \sqrt{ } 91)$ unit cells with respect to the $\mathrm{Au}(111)$ substrate. Pre-covered admantanethiol SAMs with weak van der Waals interactions can be easily displaced by alkanethiols or other thiols, which makes it possible to fabricate functional nanostructures or patterns. ${ }^{12,15}$ Cyclohexanethiols (CHTs) with six-membered cyclic rings have various structural isomers. ${ }^{16}$ Scanning tunneling microscopy (STM) and surface enhanced Raman scattering (SERS) measurements revealed that CHT SAMs have well-ordered SAMs containing two energetically stable conformers, i.e., axial and equatorial conformers. ${ }^{17,18}$ The formation and 2D structures of CHT SAMs on $\mathrm{Au}(111)$ are strongly influenced by preparation conditions, such as solution concentration and temperature, which can be attributed to the conformational dynamics of the ring. ${ }^{19,20}$ The molecular density of CHT SAMs is approximately four times less than that of alkanethiol SAMs. Therefore, CHT SAMs can be used in effective transient layers for guiding well-ordered 2D SAM growth of other thiols using the displacement technique, as 
demonstrated in previous works. ${ }^{21,22}$

Compared with several studies of CHT SAMs formed on gold, there has only been one publication describing the formation and structure of cyclopentanethiols $\left(\mathrm{C}_{5} \mathrm{H}_{9} \mathrm{SH}\right.$, CPT) with a five-membered aliphatic cyclic ring. ${ }^{23}$ Therefore, it is essential to examine these SAMs more systematically using surface characterization techniques for a better understanding of their structure and thermal stability, which can provide useful information for potential applications. In this paper, we report the first thermal desorption behaviors of CPT SAMs on $\mathrm{Au}(111)$, which were markedly different from those of simple alkanethiol SAMs, and the surface structure and adsorption conditions of the SAMs.

\section{Experimental Section}

$\mathrm{Au}(111)$ substrates with atomically flat terraces of 100 $300 \mathrm{~nm}$ in diameter were prepared by the thermal evaporation of gold onto freshly cleaved mica sheets prebaked at $330^{\circ} \mathrm{C}$ under a vacuum pressure of $10^{-5}-10^{-6} \mathrm{~Pa}$. CPT was purchased from Tokyo Chemical Industry (Tokyo, Japan) and used without further purification. CPT SAMs were prepared by immersing the $\mathrm{Au}(111)$ substrates in freshly prepared $1 \mathrm{mM}$ ethanol solutions of CPT at room temperature or $50{ }^{\circ} \mathrm{C}$ for $24 \mathrm{~h}$. Prior to surface analysis, the SAM samples were thoroughly rinsed with pure ethanol to remove physisorbed molecules from the surface and blown dry with nitrogen.

STM measurements (NanoScope E, Veeco, Santa Barbara, CA, USA) were performed in air with mechanically cut $\mathrm{Pt} / \mathrm{Ir}$ tips (80:20). STM images were recorded in the constant current mode using a sample positive bias voltage $\left(\mathrm{V}_{\mathrm{b}}\right)$ ranging from 300 to $500 \mathrm{mV}$ and a tunneling current $\left(\mathrm{I}_{\mathrm{t}}\right)$ ranging from 300 to $500 \mathrm{pA}$ between the tip and sample. XPS measurements were performed using a Theta Probe (Thermo Fisher Scientific, U.K.). Monochromated Al $\mathrm{K} \alpha$ radiation $(1486.6 \mathrm{eV})$ was used as a X-ray source. The emitted electrons were corrected at angles from 23 to $83^{\circ}$ and the spectra were calibrated from the $\mathrm{Au} 4 \mathrm{f}_{7 / 2}$ peak at $87.6 \mathrm{eV}$. TDS measurements were performed using a WA-1000S system (ESCO Ltd.) with a quadrupole mass spectrometer (QMD422; Balzers). The heating rate was approximately $1 \mathrm{~K} / \mathrm{s}$ and the vacuum pressure in the chamber was increased to $\sim 10^{-6} \mathrm{~Pa}$ during TDS measurements.

\section{Results and Discussion}

The STM image in Figure 1 shows the surface structures of CPT SAMs on $\mathrm{Au}(111)$ formed in a $1 \mathrm{mM}$ ethanol solution at room temperature or $50{ }^{\circ} \mathrm{C}$ for $24 \mathrm{~h}$. We observed considerable differences in the formation and structures of CPT SAMs depending on the solution temperature. The adsorption of CPT on $\mathrm{Au}(111)$ at room temperature generated only disordered SAMs, as shown in Figure 1(a). The formation of disordered CPT SAMs can be attributed to both weak interactions between cyclopentyl rings with a slightly distorted structure (nonplanar) and slow structural rearrange- ments. Similar structures are often observed for the SAMs of $n$-alkanethiols with short alkyl chains $\left(\mathrm{HS}\left(\mathrm{CH}_{2}\right)_{\mathrm{n}} \mathrm{CH}_{3}, \mathrm{n} \leq\right.$ $5) .{ }^{24}$ Based on these results, van der Waals interactions are considered to play crucial roles in determining the structural order of SAMs. Alternatively, our previous study showed that partially ordered CPT SAMs were formed on $\mathrm{Au}(111)$ with a $(\sqrt{ } 3.5 \times \sqrt{5}) \mathrm{R} 25^{\circ}$ structure after long-term incubation of pre-covered CPT SAMs at room temperature for $24 \mathrm{~h}^{23}$ This structural transition of CPT SAMs from the disordered to the ordered phase was attributed to the structural rearrangements of cyclopentyl rings driven by optimization of van der Waals interactions. Structural order of organic thiol SAMs can be controlled by deposition temperature, ${ }^{4,25}$ solvent property, ${ }^{7}$ and thermal annealing. ${ }^{1,6}$ It has been demonstrated that organic SAMs with a higher degree of structural order and lower structural defects were formed at elevated solution temperature compared to room temperature. STM images in Figure 1(b) and 2(a) show that the adsorption of CPT molecules at $50{ }^{\circ} \mathrm{C}$ led to the formation of well-ordered SAMs containing a number of vacancy islands (VIs). The existence of VIs with clear shapes implies that CPT molecules form crystallized SAMs via the chemical interactions between the CPT molecules and gold surfaces. From this study, we found that crystallized CPT SAMs with
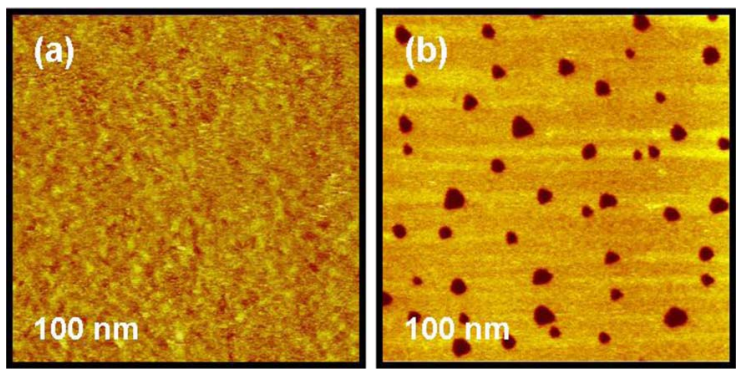

Figure 1. STM images of CPT SAMs on Au(111) formed after 24 $\mathrm{h}$ deposition in a $1 \mathrm{mM}$ ethanol solution at (a) room temperature and (b) $50{ }^{\circ} \mathrm{C}$. (a and b) Imaging parameters: $\mathrm{I}_{\mathrm{t}}=0.3 \mathrm{nA}$ and $\mathrm{V}_{\mathrm{b}}=$ $500 \mathrm{mV}$.
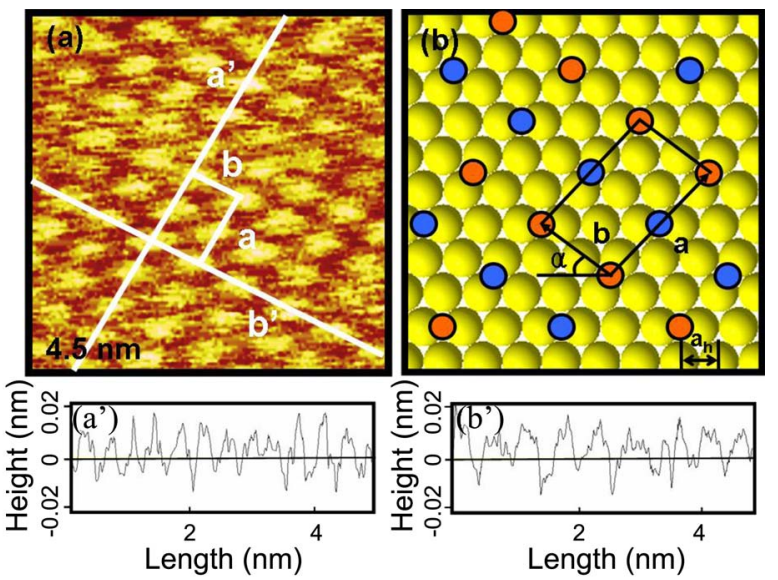

Figure 2. (a) Molecularly resolved STM image of CPT SAMs on $\mathrm{Au}(111)$ formed after $24 \mathrm{~h}$ deposition in a $1 \mathrm{mM}$ ethanol solution at $50{ }^{\circ} \mathrm{C}$. Imaging parameters: $\mathrm{I}_{\mathrm{t}}=0.38 \mathrm{nA}$ and $\mathrm{V}_{\mathrm{b}}=500 \mathrm{mV}$. (b) The proposed structural model of CPT SAMs on $\mathrm{Au}(111)$. 
a high degree of structural order could be easily obtained using a higher solution temperature of $50{ }^{\circ} \mathrm{C}$, similar to alkanethiol or aromatic thiol SAMs.

The high-resolution STM image in Figure 2(a) clearly shows closely packed molecular arrangements of CPT SAMs at $50^{\circ} \mathrm{C}$. Height profiles in Figures 2(a)' and (b)' taken along lines (a)' and (b)' on the image present superperiodicities of CPT SAMs with a high structural order. Based on this molecularly resolved STM observation, the lattice structure with an oblique unit cell contained two adsorbed molecules: $\mathrm{a}=10.3 \pm 0.2 \AA=2 \sqrt{ } 3, \mathrm{~b}=6.5 \pm 0.2 \AA=\sqrt{5}$, and $\alpha=41^{\circ}$. The surface structure of CPT SAMs can be described as a $(2 \sqrt{3} \times \sqrt{5}) \mathrm{R} 41^{\circ}$ adlayer structure, as shown in the structural model of Figure 2(b). It was assumed that all sulfur atoms occupied the bridge sites of the $\mathrm{Au}(111)$ surfaces. The observed packing structure was slightly different than that of CPT SAMs formed after long-term incubation at room temperature for $24 \mathrm{~h} .{ }^{23}$ From this study, we speculate that the formation of well-ordered CPT SAMs after a short immersion of $12 \mathrm{~h}$ at $50{ }^{\circ} \mathrm{C}$ was due to the facile movement of CPT molecules on $\mathrm{Au}(111)$ surfaces at a high surface temperature, which made it possible to facilitate the structural rearrangement of CPT SAMs.

To understand the interactions between the sulfur atoms and gold surface, XPS spectra were performed on the CPT SAMs on $\mathrm{Au}(111)$ formed at room temperature in the $\mathrm{S} 2 \mathrm{p}$ region. In general, the $S 2 p$ peaks emerge as a doublet corresponding to $2 p_{3 / 2}$ and $2 p_{1 / 2}$ peaks with an intensity ratio of 2:1 by spin-orbital splitting. ${ }^{25-27}$ Two $S 2 p_{3 / 2}$ peaks (A and $B$ ) for CPT SAMs on $\mathrm{Au}(111)$ in Figure 3 were observed at 161.2 and $162.1 \mathrm{eV}$. Both peaks can be assigned to the chemisorbed sulfurs, which are usually observed in alkanethiol $\mathrm{SAMs}^{26}$ or other organosulfur SAMs. ${ }^{25,27}$ The XPS results clearly showed that the SAMs were formed via chemical interactions between the sulfur atoms and gold surfaces. Alternatively, a physisorbed sulfur peak near 164 $\mathrm{eV}$ was not observed, suggesting that the liquid-like disordered phases of CPT SAMs at room temperature were not a result of physisorbed molecules in the SAMs. Although the

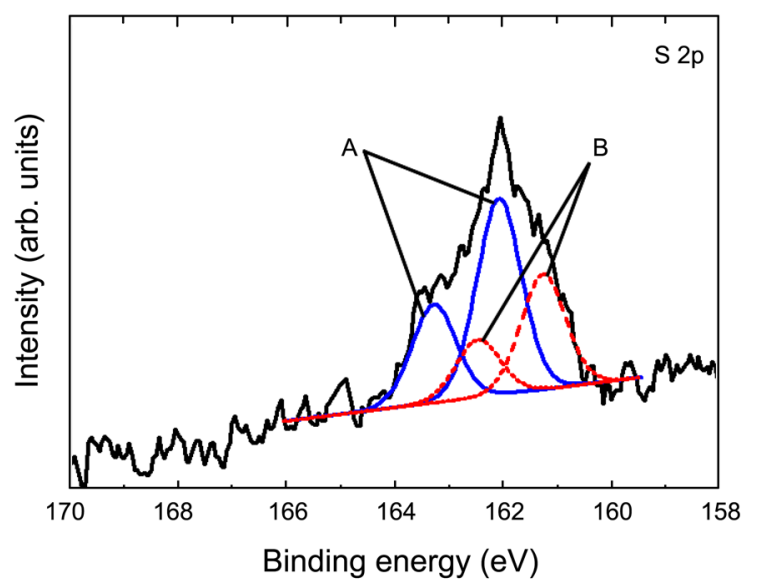

Figure 3. XPS spectra in the $S 2 p$ region for CPT SAMs on $\mathrm{Au}(111)$ formed in a $1 \mathrm{mM}$ ethanol solution at room temperature for $24 \mathrm{~h}$. strong chemisorbed sulfur peak (A) near $162.1 \mathrm{eV}$ was observed from closely packed and well-ordered alkanethiol SAMs, the relatively weak chemisorbed sulfur peak (B) near $161.2 \mathrm{eV}$ is typically observed from alkanethiol SAMs with a lower molecular density or other complicated SAM systems with a lower structural order. However, the origin of peak B remains unclear and under discussion. ${ }^{26,27}$ The formation of a disordered phase for CPT SAMs may be related to the presence of sulfur peak $\mathrm{B}$, as demonstrated by other SAM systems. ${ }^{26}$ In fact, a change in the adsorption geometry of the cyclopentyl ring can alter the hybridization condition of sulfur atoms adsorbed on gold surfaces, resulting in a change of adsorption energy of sulfurs. Therefore, it is reasonable to assume that the disordered phase of CPT SAMs was due to an inhomogeneous adsorption geometry, as suggested by the coexistence of two different chemisorbed sulfur atoms.

To examine thermal desorption behaviors and stability of CPT SAMs on $\mathrm{Au}(111)$, we monitored desorption species from the SAMs as a function of the surface temperature. TD spectra for CPT SAMs in Figure 4 show two dominant desorption peaks for the $\mathrm{C}_{5} \mathrm{H}_{9}^{+}(\mathrm{m} / \mathrm{e}=69)$ and $\mathrm{C}_{5} \mathrm{H}_{9} \mathrm{SH}^{+}$ $(\mathrm{m} / \mathrm{e}=102)$ species. TD spectra with a very strong intensity corresponding to the cyclopentyl ring fragments $\left(\mathrm{C}_{5} \mathrm{H}_{9}{ }^{+}\right)$ formed via $\mathrm{C}-\mathrm{S}$ bond cleavage were observed near $440 \mathrm{~K}$. These decomposed species were initially desorbed at $340 \mathrm{~K}$. Another desorption peak with a relatively strong intensity corresponding to the parent molecular species $\left(\mathrm{C}_{5} \mathrm{H}_{9} \mathrm{SH}^{+}\right)$ was also observed at $440 \mathrm{~K}$. Although XPS results showed no physisorbed species from CPT SAMs, parent mass desorption species was observed. It has been reported that the origin of these desorption species are due to a recombination of the chemisorbed thiolates and hydrogen atoms, which may be derived from the thermal decomposition of adsorbed molecules during heating and/or a hydrogen abstraction reaction of thiolates from the walls inside chamber. ${ }^{9,28,29}$ In addition, TD spectra with a very weak intensity for chemi-

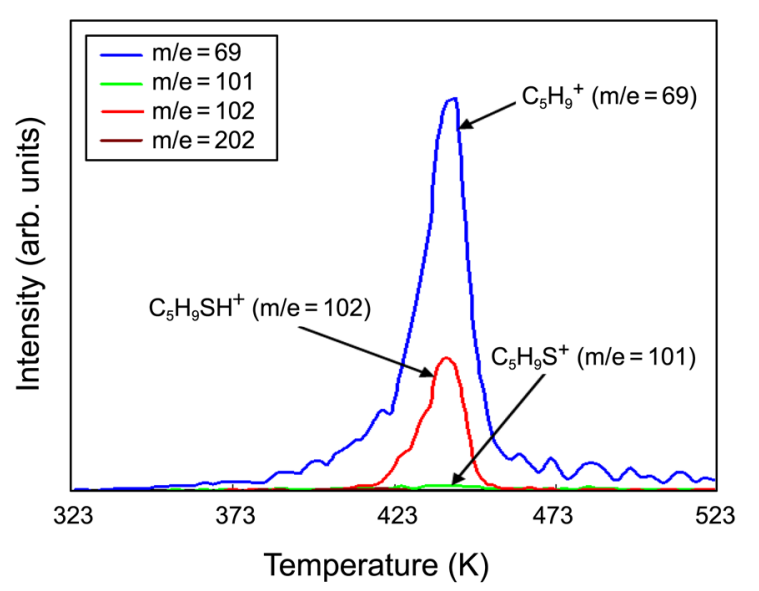

Figure 4. TDS spectra of CPT SAMs on $\mathrm{Au}(111)$ formed in a 1 $\mathrm{mM}$ ethanol solution at room temperature for $24 \mathrm{~h}$. Three desorption peaks near $440 \mathrm{~K}$ were observed for the decomposed cyclopentyl species $\left(\mathrm{C}_{5} \mathrm{H}_{9}^{+}\right)$, parent mass species $\left(\mathrm{C}_{5} \mathrm{H}_{9} \mathrm{SH}^{+}\right)$, and chemisorbed species $\left(\mathrm{C}_{5} \mathrm{H}_{9} \mathrm{~S}^{+}\right)$. 


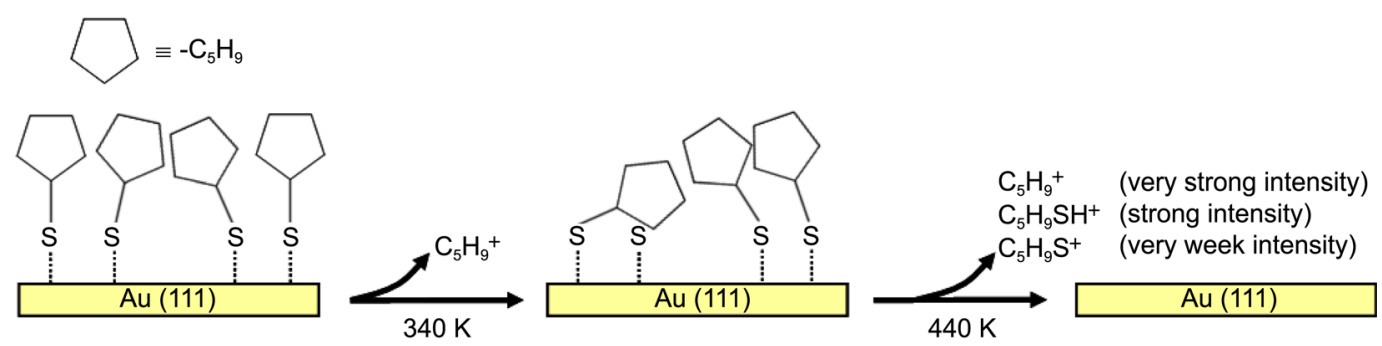

Figure 5. A schematic showing thermal desorption behaviors of CPT SAMs on Au(111).

sorbed thiolate species $\left(\mathrm{C}_{5} \mathrm{H}_{9} \mathrm{~S}^{+} ; \mathrm{m} / \mathrm{e}=101\right)$ were observed at $440 \mathrm{~K}$. Three desorption fragments with a maximum intensity appeared near $440 \mathrm{~K}$. Based on the XPS and TDS results, the strong decomposed species $\left(\mathrm{C}_{5} \mathrm{H}_{9}{ }^{+}\right)$resulted from preferential $\mathrm{C}-\mathrm{S}$ bond cleavage compared to $\mathrm{S}-\mathrm{Au}$ bond cleavage from chemisorbed cyclopentanethiolate SAMs on the Au(111) surface. Alternatively, $n$-alkanethiol SAMs at saturated coverage typically show strong TD spectra of dialkyl disulfide species as a result of associative desorption of alkanethiolates, in addition to strong TD spectra of parent molecular species and alkanethiolate species. ${ }^{9,29}$ However, we did not observe dicyclopentyl disulfide species $\left(\mathrm{C}_{5} \mathrm{H}_{9} \mathrm{~S}\right.$ $\mathrm{SC}_{5} \mathrm{H}_{9}{ }^{+}, \mathrm{m} / \mathrm{e}=202$ ) for CPT SAMs on $\mathrm{Au}(111)$. This result suggests that the dimerization of sulfur headgroups in CPT SAMs on $\mathrm{Au}(111)$ during heating was difficult because of the bulkiness of the tail groups of the CPT molecules, which can significantly suppress associative desorption. The SAMs of CHT molecules with cyclohexyl rings also did not show dimerization reactions due to steric hindrance of their bulky tail groups. ${ }^{9}$ Based on the TDS study, tail groups with alicyclic ring systems markedly inhibited the associative dimerization process of sulfur headgroups during heating. Figure 5 shows a schematic of the desorption processes of CPT SAMs on $\mathrm{Au}(111)$ during heating. The thermal desorption behaviors and stability of SAMs of CPT with an alicyclic ring on $\mathrm{Au}(111)$ were also markedly different from those of SAMs of $n$-alkanethiol with a normal alkyl chain.

\section{Conclusions}

CPT SAMs formed at room temperature on $\mathrm{Au}(111)$ with disordered phases, whereas CPT SAMs formed at $50{ }^{\circ} \mathrm{C}$ were $2 \mathrm{D}$ well-ordered SAMs that could be described as $(2 \sqrt{3}$ $\times \sqrt{5}) \mathrm{R} 41^{\circ}$ in structure. In this study, solution temperature was one of the most important parameters for controlling surface structure and the structural order of CPT SAMs on $\mathrm{Au}(111)$. XPS measurements for CPT SAMs at room temperature revealed the presence of two $S 2 p_{3 / 2}$ peaks at 161.2 and $162.1 \mathrm{eV}$, suggesting that the SAMs were formed via chemical reactions between the sulfur atoms and $\mathrm{Au}(111)$ surfaces. We observed two dominant TD peaks for decomposed fragments $\left(\mathrm{C}_{5} \mathrm{H}_{9}{ }^{+}\right)$generated via $\mathrm{S}-\mathrm{C}$ bond cleavage and the parent molecular species $\left(\mathrm{C}_{5} \mathrm{H}_{9} \mathrm{SH}^{+}\right)$derived from recombination of the chemisorbed thiolates and hydrogen atoms at around $440 \mathrm{~K}$. Although dimerization of sulfur atoms in $n$ alkanethiol SAMs usually occurs during thermal desorption, the dimerization reaction for CPT SAMs was not observed, which may be due to suppression by the steric hindrance of the cyclic ring of CPT molecules. In this study, we clearly demonstrated that the alicyclic rings of organic thiols strongly affect the surface structures and thermal desorption behaviors of SAMs.

Acknowledgments. This research was supported by a grant from the Korea Research Foundation (KRF) funded by the Korean Government (KRF-2008-313-C00410) and the International Research \& Development Program (K2090100000609E0100-00610) through the National Research Foundation of Korea funded by the Ministry of Education, Science and Technology, the Seoul R\&BD Program (10919), and the research fund of Hanyang University (HYU-2011-T).

\section{References}

1. Love, J. C.; Estroff, L. A.; Kriebel, J. K.; Nuzo, R. G.; Whitesides, G. M. Chem. Rev. 2005, 105, 1103.

2. Schreiber, F. J. Phys.: Condens. Matter 2004, 16, R881.

3. Vericat, C.; Vela, M. E.; Benitez, G.; Carro, P.; Salvarezza, P. Chem. Soc. Rev. 2010, 39, 1805.

4. Kang, H.; Lee, N.-S.; Ito, E.; Hara, M.; Noh, J. Langmuir 2010 , $26,2983$.

5. Noh, J.; Hara, M. Langmuir 2002, 18, 1953.

6. Noh, J.; Kato, H. S.; Kawai, M.; Hara, M. J. Phys. Chem. B 2006, 110, 2793.

7. Lee, N.-S.; Kang, H.; Ito, E.; Hara, M.; Noh, J. Bull. Korean Chem. Soc. 2010, 31, 2137.

8. Choi, Y.; Choi, I.; Kang, H.; Cho, J.-H.; Jang, C.-H.; Noh, J. Bull. Korean Chem. Soc. 2010, 31, 904.

9. Hayashi, T.; Wakamatsu, K.; Ito, E.; Hara, M. J. Phys. Chem. C 2009, 113, 18795.

10. Chesneau, F.; Zhao, J.; Shen, C.; Buck, M.; Zharnikov, M. 2010, 114,7112

11. Dameron, A. A.; Charles, L. F.; Weiss, P. S. J. Am. Chem. Soc. 2005, 127, 8697.

12. Kim, M.; Hohman, J. N.; Morin, E. I.; Daniel, T. A.; Weiss, P. S. J. Phys. Chem. A 2009, 113, 3895.

13. Hohman, J. N.; Zhang, P.; Morin, E. I.; Han, P.; Kim, M.; Kurland, A. R.; McClanahan, P. D.; Balema, V. P.; Weiss, P. S. ACS Nano 2009, 3, 527.

14. Fujii, S.; Akiba, U.; Fujihira, M. J. Am. Chem. Soc. 2002, 124, 13629.

15. Dameron, A. A.; Hampton, J. R.; Smith, R. K.; Mullen, T. J.; Gillmor, S. D.; Weiss, P. S. Nano Lett. 2005, 5, 1834.

16. Kwon, C. K.; Kim, M. S.; Kim, K. J. Raman Spectrosc. 1989, 20 , 575.

17. Noh, J.; Hara, M. Langmuir 2001, 17, 7280.

18. Joo, S. W.; Chung, H.; Kim, K.; Noh, J. Surf. Sci. 2007, 601, 3196.

19. Kwon, S.; Jeong, Y.; Lee, Y.; Noh, J. Chem. Lett. 2007, 36, 307. 
20. Kang, H.; Jang, C.-H.; Hara, M.; Noh, J. J. Nanosci. Nanotechnol. 2009, 9, 7085 .

21. Kang, H.; Lee, H.; Kang, Y.; Hara, M.; Noh, J. Chem. Commun. 2008, 5197.

22. Kwon, S.; Choi, Y.; Choi, J.; Kang, H.; Chung, H.; Hara, M.; Noh, J. Ultramicroscopy 2008, 108, 1311.

23. Noh, J. Bull. Korean Chem. Soc. 2006, 27, 944.

24. Poirier, G. E.; Tarlov, M. J.; Rushmeier, H. E. Langmuir 1994, 10, 2883
25. Choi, Y.; Jeong, Y.; Chung, H.; Ito, E.; Hara, M.; Noh, J. Langmuir 2008, 24,91 .

26. Ishida, T.; Hara, M.; Kojima, M.; Tsuneda, S.; Nishida, N.; Sasabe, H.; Knoll, W. Langmuir 1998, 14, 2092.

27. Noh, J.; Jeong, Y.; Ito, E.; Hara, M. J. Phys. Chem. C 2007, 111, 2691.

28. Noh, J.; Ito, E.; Hara, M. J. Colloid Interface Sci. 2010, 342, 513.

29. Kondo, H.; Komada, C.; Nozoye, H. J. Phys. Chem. B 1998, 102, 2310 . 